\title{
An Exploratory Study of Virtual Fit Testing using 3D Virtual Fit Models and Garment Simulation Technology in Technical Design
}

\author{
MyungHee SOHN*, Lushan SUN \\ University of Missouri, Columbia MO, USA \\ http://dx.doi.org/10.15221/13.067
}

\begin{abstract}
The use of Three-Dimensional (3D) body scanning technology in the area of technical apparel design has begun to receive more attention and be implemented in the industry over the recent decade. The purpose of this study was to observe the effectiveness of $3 D$ virtual fit testing processes using body scans and garment simulation technology and to identify the areas of challenge in the process of 3D virtual fit and pattern manipulation. In this study, two fit models were recruited based on ASTM measurements for Misses and Women's plus size. One Misses size 8 model represented the standard size as a control, and the other model was Women's plus-sized 16. Fit models were scanned and created into 3D virtual models (VM) using a $[\mathrm{TC}]^{2}$ body scanner. Two types of garments were selected as test garments: a basic unlined bodice and pants. Both garments were made from a medium-weight cotton woven fabric. These garments were selected for their representations of basic garment style, and there are definite expectations regarding the fit. Three advanced undergraduate apparel design students were selected and trained. Each participant manipulated the patterns to fit each virtual model (VM). The virtual fit testing and fit correction were recorded using Camtasia screen recording software. Then, the actual garments were made and tested on live models. Live fit models evaluated the comfort and fit of the garments. The results indicated that participants spent more time on size 16 than size 8 , and the bodice took twice as much time as the pants. Based on the size 8 fit model evaluation, the least satisfying fit was found in the neckline, crotch, and pant hem. This may be due to the difficulty in determining fitting ease using 3D simulation. The results of the size 16 live fit test showed that poor fit was found in the underarm, neckline, abdomen, buttocks, hip girth, and thigh. More fit problems on the pants were found in size 16. This may be due to lack of knowledge among participants concerning plus-size pants fit and pattern shape. This study revealed that fit ease and certain areas of greater body curve should be further tested on the live fit model for accuracy.
\end{abstract}

Keywords: 3D body scanning technology, 3D virtual fit, technical design

\section{Introduction}

3D body scanning technology has been used in a variety of industries, including movie animation for special effects and ergonomic shape design in car production [1]. In the apparel industry, this technology has been used to obtain accurate body measurements for body/apparel sizing and pattern grading. As 3D body scanning technology becomes more sophisticated and widely implemented worldwide, some systems have already been made commercially available. The ability to present garments via 3D graphics has been an innovative approach to assessing and visualizing apparel designs and fit more efficiently in today's apparel industry. Fit problems still exist in various stages of apparel design, especially in the pattern grading and sizing system [2]. Having a wide variety of fit standards, women's apparel requires a great deal of time and labor in the fit testing sessions. However, due to the benefit of the $3 D$ body scanning technology and $3 D$ virtual simulation, it is possible to test garment patterns virtually on the virtual fit model in a much more time-efficient manner. The body scan can be used for 3D simulation as a virtual fit model. This would be useful in assessing adequate fit for the targeted consumer [3].

Studies on virtual fit testing using 3D simulation technology are currently limited, and the reliability and validity of virtual fit analysis are still being debated. Therefore, this study specifically focused on 3D virtual fitting when using 3D body scanning technology and 3D virtual simulation to examine pattern manipulation through comparing virtual and live fitting sessions. For this research, a 3D body scanning system, $[\mathrm{TC}]^{2}$, was used to obtain a body scan and create a virtual model.

\subsection{D CAD system integration}

Parallel to the development of 3D body scanning technology, 3D Computer-Aided-Design (CAD) software has also been created to support the efficiency in apparel product development. Software such as Optitex, Browzwear, and PAD System Technologies aid the process through draping 2D patterns over 3D avatars developed from body scan data [1]. Some even allow the designer to manipulate patterns three-dimensionally and flatten back into two dimensions for further production [1].

*sohnm@missouri.edu; +1 573-882-6410; tam.missouri.edu 
Generally, to produce a garment traditionally, the average model goes through 2-3 fitting sessions that last 1-2 hours each [3]. Fit sessions are held an average 4-15 times per season, depending on the company. These sessions translate into added costs of personnel, fit models, and product development. This system has been widely adopted in apparel companies including Target, Kohl's, Patagonia, and Wal-mart. Companies such as Victoria's Secret also use video and Internet conferencing to conduct fitting sessions on both fit models and dress forms from remote locations [4]. With the use of 3D CAD systems, companies would be able to reduce the cost of product development by eliminating the amount of time and labor spent on fitting sessions on live fit models or dress forms.

The 3D body scanning technology enables a quick and consistent body measurement extraction, and the use of virtual fitting simulation systems further enables such technology to aid the processes of the apparel industry. It is essential for such application to be practiced with knowledge of accurate combinations of both $3 \mathrm{D}$ and $2 \mathrm{D}$ garment images, which requires sensitive detection of body scan data and cloth [5].

\subsection{Fit in apparel product development process}

Apparel fit has been defined by various researchers and companies in the past based on one's perception on the lifestyle and culture. In the United States, sizing standard is challenged by the population of diverse body type, and obtaining accurate physical measurement is essential in developing the appropriate fit for a specific body type, especially for women. Generally, fit can be defined through factors of ease, line, grain, balance, and set [6]. The traditional way of measuring method in apparel product development is usually the linear method, in which only the length of chosen areas of the body is collected and utilized in pattern development using tape measure [7]. Not only is it difficult for the process to achieve accuracy in fit, causing product dissatisfaction that rate $60 \%$ among female consumers, but also is less desirable and embarrassing for the some women due to unavoidable touch [8]. For plus-sized women, such measurements are hard to standardize and considered unreliable due to the inframammary fold [8]. Today, some companies, such as David's Bridal, adapts the fit model "double", dress form with more defined curvature in attempt to improve fitting accuracy and production efficiency but still does not solve the problem [4]. Fitting issues would also result from the multiple fitting sessions conducted offshore locations around the world due to the barrier in inappropriate communication environment and consistency in prototype development standard between the design company and production vendor [9]. On the other hand, non-contact 3D body scanning offers the convenience of collecting extensive anthropometric data in seconds with convertible true-to-scale avatar form. Today, 3D body scanning technology serves as the bridge between product quality and CAD technologies [9].

Question still remain in how accurate is virtual fit process? What specific areas of the garment do students have difficulties in correcting misfit, and why? On which part of the fit-analysis process do the participants spend the most time, and why? The objective of this study was to assess the effectiveness of $3 \mathrm{D}$ virtual fit testing process through comparing virtual fit to real fit produced by student participants, and to identify the area of challenge for each participant in the process of $3 \mathrm{D}$ virtual fit and pattern manipulation.

\section{Method}

The case study method was used because it is believed to be useful in human affairs study under natural contexts. It was selected to thoroughly consider the user's experience and the entire process $[10,11]$. This study, based on the case study approach, replicated a process of fit testing and pattern manipulation in technical design. Technical designers can test garment fit on a virtual fit model developed from the live fit model and manipulate pattern pieces to fit the virtual fit model. Then, they can compare the virtual fit to actual fit by producing actual garments.

\subsection{Data collection and procedure}

\subsubsection{Participants}

Two fit models were selected through the screening process, based on ASTM measurements for Misses and Women's plus-size (Table 1). One Misses size 8 fit model was used to represent the standard size as a control, and the other model selected represents Women's plus-size 16. Two fit models were asked to visit two times for body scanning and live fit testing. Three advanced undergraduate apparel design students participated in this study to complete processes of digital pattern manipulation, virtual fitting tests, prototype development, and live fitting tests. They were selected based on their understanding in the concept and skills of garment fit and virtual fit testing and 
pattern manipulation using 3D CAD software, OptiTex ${ }^{\circledR}$. These students were enrolled in a class in technical design and virtual fit. Institutional Review Board approval was acquired before proceeding with the study.

Table 1. ASTM measurements for misses size 8 and women's plus-size 16.

\begin{tabular}{|c|c|c|}
\hline & Misses size 8 & Women plus size 16 \\
\hline Bust & $35^{\prime \prime}$ & $41.5^{\prime \prime}$ \\
\hline Waist & $27 "$ & $33.5^{\prime \prime}$ \\
\hline Hip & $37.5^{\prime \prime}$ & $43.5^{\prime \prime}$ \\
\hline
\end{tabular}

\subsubsection{Test garments}

An unlined, sleeveless, collarless basic top and pants with no pocket, made of medium-weight cotton woven fabric were selected as test garments. Misses size 8 basic bodice pattern with one waist dart and two waist darts patterns were manipulated to fit two models. They were selected because participants were familiar with these basic patterns and would have a definite expectation regarding the fit of basic garments.

\subsubsection{Procedure}

Misses size 8 and Women's plus-size 16 fit models were scanned in their everyday underwear using a 3D body scanner. Their body scans were converted into 3D virtual fit models from KX-16 body scanner software. Each student participant was instructed to manipulate the one set of basic patterns fit two different model in the 2D/3D pattern making software, OptiTex ${ }^{\circledR}$. Previously created Size 8 and Size 16 virtual fit models were utilized in the digital pattern manipulation through visualizing 3D simulation (Figure 1). The participants visually evaluated the virtual garment fit based on elements of ease, line, balance, set and grain [1]. The entire virtual fitting processes were documented through computer monitor recording using Camtasia ${ }^{\circledR}$. Finalized patterns were printed to true scale and used to produce prototype garments in medium-weight cotton woven fabric to further examine the fit on the live fit models. During the fitting session, student participants were asked to note the fitting issues in the garment through the evaluation from the live fit models.

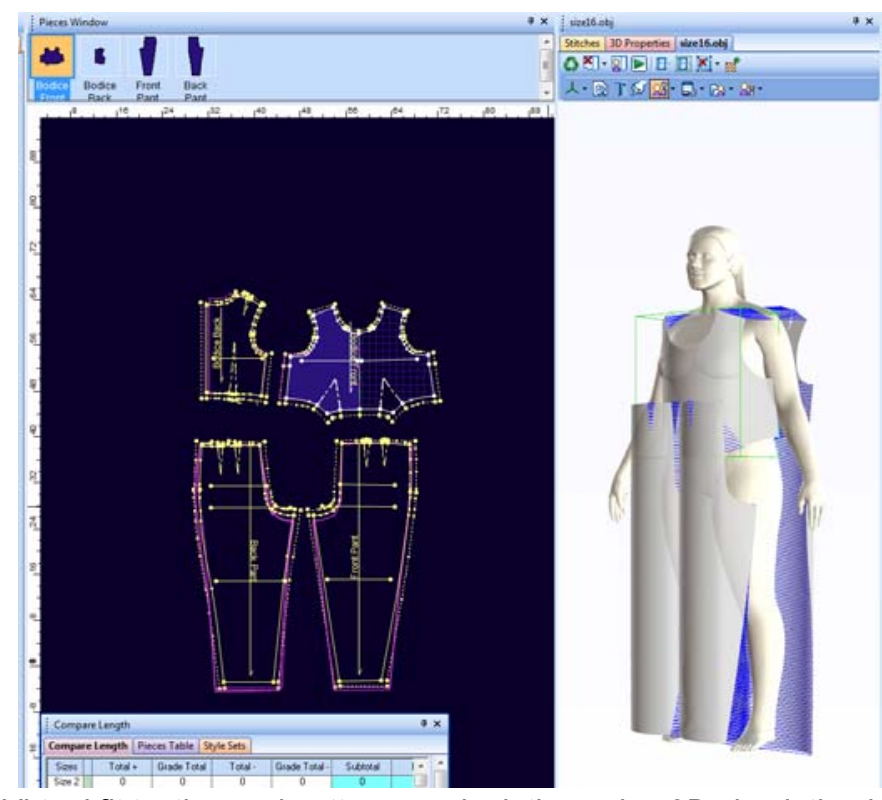

Figure 1. Virtual fit testing and pattern manipulation using $3 D$ simulation in OptiTex ${ }^{\circledR}$.

\subsection{Data analysis}

Content analysis was used to investigate the entire virtual fit testing process using garment simulation technology and to identify the area of challenge in the process of $3 \mathrm{D}$ virtual fit and pattern manipulation. Data in this case study were analyzed using the coding software for monitor recording, Interact ${ }^{\circledR}$, to determine the frequency and duration of each action performed by each participant. Fit evaluation was also analyzed through the fit models' comfort and tightness level of test garments.

In analyzing the participants' computer monitor recording completed in the 3D CAD software, activities were categorized into bodice pattern manipulation, pants pattern manipulation, 3D virtual simulation 
tool application, and other actions that included participants' time of looking for tools in the software. Each activity was analyzed based on its frequency and duration ( $\mathrm{min}$ ) throughout the entire monitor recording session for each pattern size. Under bodice pattern manipulation, actions were grouped into 12 areas: front armhole change at side seam (SS-AH-F), back armhole change at side seam (SS-AHB), front waist-bust change at side seam (SS-WB-F), back waist-bust change at side seam (SS-WB$B)$, neckline (NK), front hem (H-F), back hem (H-B), shoulder (S), dart (D), front armhole (AH-F), back armhole (AH-B), and center (C). Isolated areas were divided to closely observe participants' manipulation strategy and overall change in pattern (Table 2). For example, the participants' actions in adjusting the bodice front armhole would consequently affect the length or angle of the side seam (SS-AH-F).

The pant pattern manipulation activities were grouped into 11 areas, including front waist change at pant side seam (PSS-W-F), back waist change at pant side seam (PSS-W-B), front hip curve change at pant side seam (PSS-HP-F), back hip curve change at pant side seam (PSS-HP-B), hip curve change at pant side seam (PSS-HC), pant hem (PH), front crotch (CR-F), back crotch (CR-B), pant dart (PD), inseam (IN), and waist (W). For the PSS-HP-F area, a participant would adjust the front hip edge of the pant pattern, which would at the same time result in a length or width measurement change in the pants' side seam. On the other hand, PSS-HC-F would mean the front hip curve of the pant pattern was adjusted to also cause shift in the pant side seam edge of the pattern.

In addition, live fit was evaluated by a questionnaire consisting of two sections for each of 8 critical body fit locations for the bodice (bust girth, cross chest, cross back, underarm area, neck front, neck back, neck side, waist girth, and length) and 6 for the pants (waist girth, abdomen, buttocks, hip girth, thigh, crotch, and length). Comfort level was rated on a scale of 1 to 5 , with 5 being the most comfortable. Tightness-looseness was rated on a scale of 1 to 5 : the middle value of the scale, 3 , represented good fit. The tightness of clothing was evaluated on levels of very tight, somewhat tight, good, somewhat loose, and very loose.

\section{Results}

\subsection{Descriptive analysis}

On average, the time all three participants spent on manipulating size 8 patterns ( 90.78 minutes) was less than the time spent on size 16 (98.52 minutes), and the time spent on the bodice pattern was much greater than the pant pattern, especially for size 16 (Table 2). Also, the time spent in applying the 3D simulation tool was about half of the total time used in the virtual fitting process for both size 8 and 16 patterns. Participant $\mathrm{C}$ spent more time and showed higher frequency in manipulation than the other two participants.

Table 2. Participant' 3D virtual fitting screen activity analysis by pattern manipulation action in frequency (duration).

\begin{tabular}{|c|c|c|c|c|c|c|}
\hline \multicolumn{7}{|c|}{ Participants' 3D Virtual Fitting Screen Activity (frequency and duration in minute) } \\
\hline & \multicolumn{2}{|c|}{ Participant A } & \multicolumn{2}{|c|}{ Participant B } & \multicolumn{2}{|c|}{ Participant C } \\
\hline Pattern Area & Size 8 & Size 16 & Size 8 & Size 16 & Size 8 & Size 16 \\
\hline \multicolumn{7}{|c|}{ Bodice } \\
\hline SS-AH-F & na & $5(0.74)$ & $1(0.33)$ & $6(1.23)$ & na & $33(4.03)$ \\
\hline SS-AH-B & $3(1.52)$ & $1(0.04)$ & na & $1(0.71)$ & na & $9(1.27)$ \\
\hline SS-WB-F & $7(2.96)$ & $8(1.84)$ & $8(1.90)$ & $7(2.36)$ & $1(0.12)$ & $16(2.68)$ \\
\hline SS-WB-B & $7(3.06)$ & $6(0.80)$ & na & $1(0.34)$ & $1(0.09)$ & $5(1.29)$ \\
\hline NK & $2(0.53)$ & $4(0.45)$ & $6(4.43)$ & $9(3.79)$ & $11(2.00)$ & $6(0.89)$ \\
\hline $\mathrm{H}-\mathrm{F}$ & $2(0.72)$ & 8 (1.43) & $8(2.27)$ & $4(1.02)$ & $3(0.56)$ & $8(1.68)$ \\
\hline $\mathrm{H}-\mathrm{B}$ & $7(3.32)$ & $1(0.12)$ & $2(0.68)$ & $1(0.34)$ & na & $10(1.43)$ \\
\hline$S$ & na & na & na & $1(0.20)$ & $6(1.86)$ & $21(2.74)$ \\
\hline $\mathrm{D}$ & $1(1.47)$ & $12(2.84)$ & $7(2.65)$ & 18 (7.69) & $3(1.69)$ & $21(4.07)$ \\
\hline $\mathrm{AH}-\mathrm{F}$ & na & $6(0.96)$ & na & $2(0.81)$ & $7(1.09)$ & $38(6.65)$ \\
\hline $\mathrm{AH}-\mathrm{B}$ & $8(2.69)$ & na & na & $1(0.61)$ & $16(3.01)$ & $7(1.21)$ \\
\hline C & $3(0.97)$ & $3(0.22)$ & $1(0.33)$ & $11(3.72)$ & $3(0.05)$ & $4(0.60)$ \\
\hline Total Bodice & $40(17.24)$ & $54(9.44)$ & $33(12.59)$ & $62(22.82)$ & $51(10.47)$ & $178(27.94)$ \\
\hline
\end{tabular}




\begin{tabular}{|c|c|c|c|c|c|c|}
\hline \multicolumn{7}{|c|}{ Pants } \\
\hline PSS-W-F & $2(0.35)$ & $2(0.29)$ & na & $4(1.06)$ & $4(1.07)$ & $3(1.13)$ \\
\hline PSS-W-B & $3(0.86)$ & $4(0.42)$ & na & $4(1.76)$ & $3(0.49)$ & $2(0.32)$ \\
\hline PSS-HP-F & na & na & $2(1.11)$ & $1(0.25)$ & $1(0.09)$ & na \\
\hline PSS-HP-B & na & na & $2(0.89)$ & na & na & na \\
\hline PSS-HC & na & $9(1.19)$ & na & $1(0.22)$ & na & na \\
\hline $\mathrm{PH}$ & $2(1.21)$ & $5(0.38)$ & na & $2(0.84)$ & $9(0.89)$ & $3(0.29)$ \\
\hline CR-F & $2(1.79)$ & $2(0.20)$ & $19(8.41)$ & $4(1.65)$ & $4(0.65)$ & $3(0.29)$ \\
\hline CR-B & $2(0.60)$ & $2(0.45)$ & $2(0.76)$ & $1(0.66)$ & $1(0.09)$ & $2(0.33)$ \\
\hline PD & $5(2.47)$ & $5(0.82)$ & na & $1(0.17)$ & na & na \\
\hline IN & na & na & na & $5(1.72)$ & na & $5(0.53)$ \\
\hline w & $1(0.51)$ & na & na & na & 7 (1.39) & $2(0.48)$ \\
\hline Total Pants & $17(7.97)$ & $29(3.75)$ & $25(11.17)$ & $22(8.33)$ & $29(4.97)$ & $20(3.37)$ \\
\hline \multicolumn{7}{|c|}{ 3D Virtual Simulation } \\
\hline & $35(71.08)$ & $51(50.48)$ & $41(53.89)$ & $53(70.86)$ & $60(35.41)$ & $87(52.16)$ \\
\hline \multicolumn{7}{|c|}{ Other Action } \\
\hline & $14(11.99)$ & $24(18.73)$ & $15(14.25)$ & $16(17.95)$ & $29(21.32)$ & $26(9.75)$ \\
\hline Total & $106(108.28)$ & $158(82.4)$ & $114(91.90)$ & $154(119.96)$ & $169(72.17)$ & $290(93.22)$ \\
\hline
\end{tabular}

Note: SS-AH-F (front armhole change at side seam), SS-AH-B (back armhole change at side seam), SS-WB-F (front waist-bust change at side seam), SS-WB-B (back waist-bust change at side seam), NK (neckline), $H-F$ (front hem), H-B (back hem), S (shoulder), D (dart), AH-F (front armhole), AH-B (back armhole), C (center), PSS$W-F$ (front waist change at pant side seam), PSS-W-B (back waist change at pant side seam), PSS-HP-F (front hip curve change at pant side seam), PSS-HP-B (back hip curve change at pant side seam), PSS-HC (hip curve change at pant side seam), $P H$ (pant hem), CR-F (front crotch), CR-B (back crotch), PD (pant dart), IN (inseam), $W$ (waist).

Figure 2 shows the frequency of pattern manipulations from the three participants. The results show the most altered areas on the size 8 pattern included front waist-bust change at side seam, neckline, front hem, back hem, dart, and back armhole in the bodice pattern (SS-WB-F, NK, H-F, H-B, D, AH-B), and front waist at side seam, back waist at side seam, hem, front crotch, dart, and waistline in the pant pattern (PSS-W-F, PSS-W-B, PH, CR-F, PD, W). For size 16 pattern manipulation, the most altered areas included front armhole at side seam, front waistline-bust at side seam, neckline, front hem, shoulder, dart, and front armhole in bodice pattern (SS-AH-F, SS-WB-F, NK, H-F, S, D, AH-F) and front waist change at pant side seam, back waist change at side seam, hem, and front crotch in pant pattern (PSS-W-F, PSS-W-B, PH, CR-F).

Note: SS-AH-F (front armhole change at side seam), SS-AH-B (back armhole change at side seam), SS-WB-F (front waist-bust change at side seam), SS-WB-B (back waist-bust change at side seam), NK (neckline), $H-F$ (front hem), $H-B$ (back hem), S (shoulder), D (dart), AH-F (front armhole), AH-B (back armhole), $C$ (center), PSS$W-F$ (front waist change at pant side seam), PSS-W-B (back waist change at pant side seam), PSS-HP-F (front hip curve change at pant side seam), PSS-HP-B (back hip curve change at pant side seam), PSS-HC (hip curve change at pant side seam), PH (pant hem), CR-F (front crotch), CR-B (back crotch), PD (pant dart), IN (inseam), $W$ (waist). 


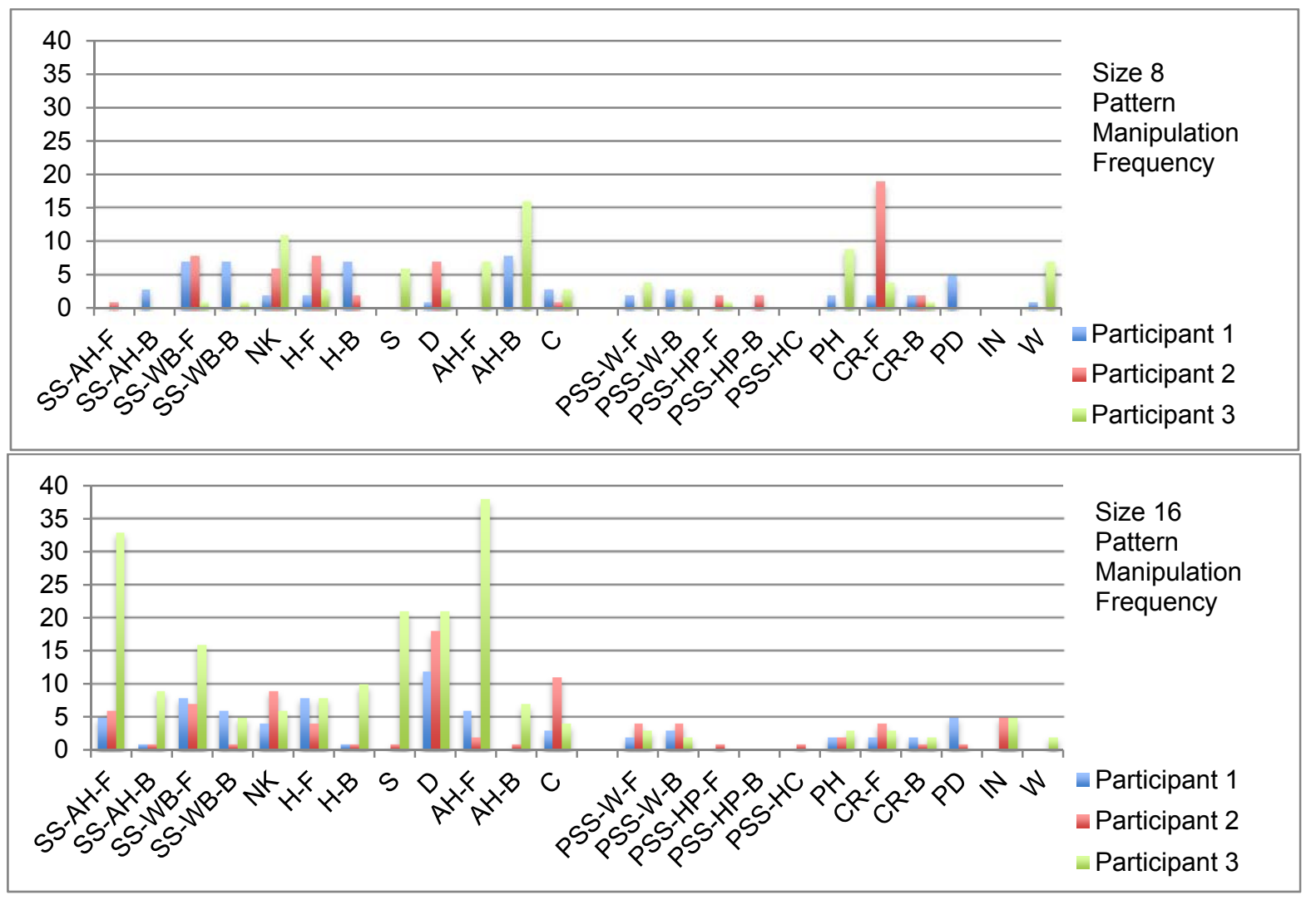

Figure 2. Participants' pattern manipulation frequency of the bodice and pant patterns for size 8 and 16 during virtual fitting session.

\subsection{Final adjusted pattern}

The result of the manipulated patterns showed that they differed in proportion changes between size 8 and 16 (Figure 3). For size 8 patterns, the three participants' manipulation resulted in somewhat similar amounts of change while size 16 patterns showed obvious differences in the measurement adjustment in the same areas in the patterns.
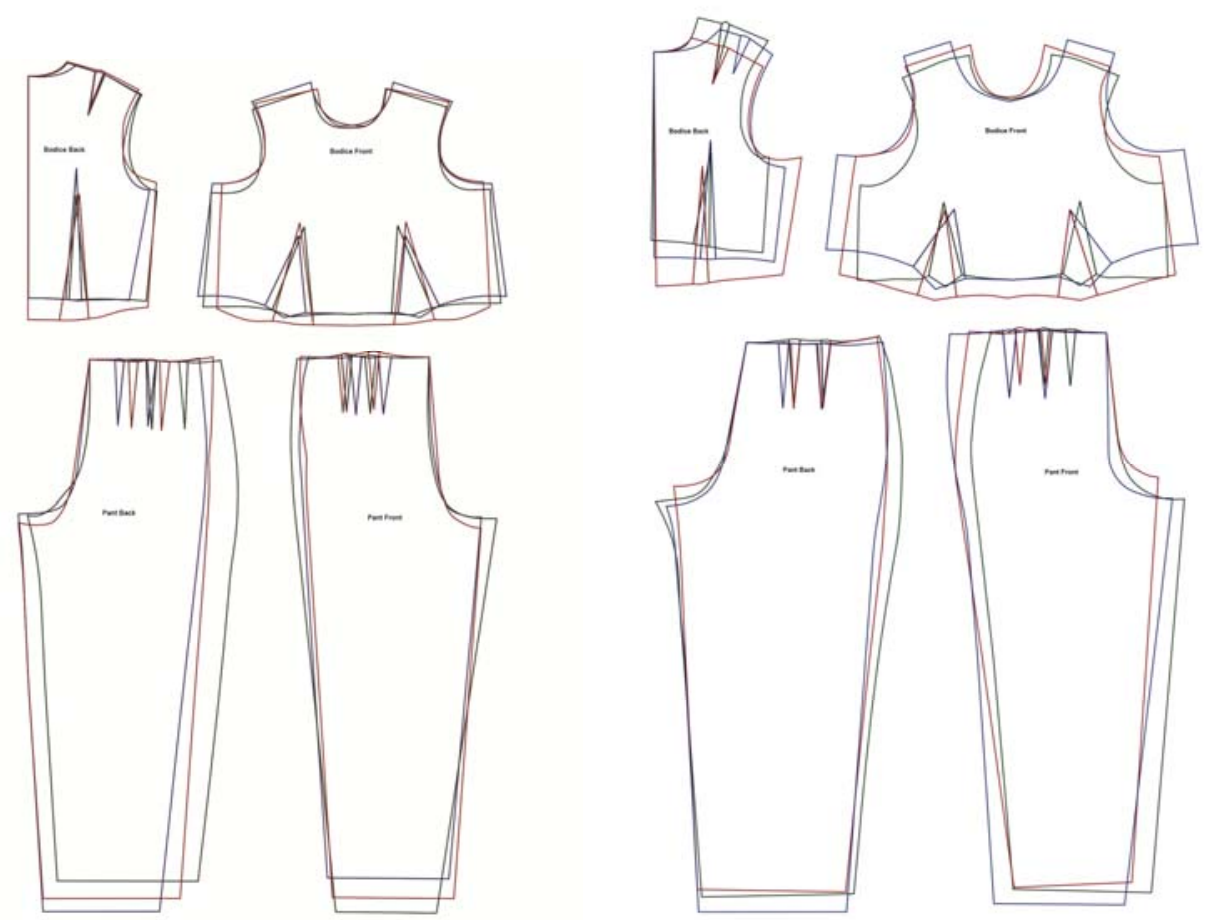

Figure 3. Manipulated bodice and pant patterns for size 8 (left) and 16 (right) using OptiTex® in order of participants 1, 2, and 3 from left to right. 
Further, both size 8 and 16 patterns depicted more manipulation in areas where high frequency of change occurred. The areas of most apparent difference existed in front waist-bust change at side seam, neckline, both front and back hem, and dart and back armhole (SS-WB-F, NK, H-F, H-B, D, $\mathrm{AH}-\mathrm{B}$ )in the bodice, and front and back waist change at pant side seam, front crotch, and pant hem (PSS-W-F, PSS-W-B, PH, CR-F) in the pant. One difference of pattern manipulation area between the two sizes was that size 16 had more adjustment and variation at the shoulder than size 8 .

\subsection{Live fit evaluation}

For this study, prototype garments were developed by the student participants from the 3D virtual fitting process and evaluated by the fit models. The results of the size 8 live fit test showed that the least fit satisfaction was found in the neckline, crotch, and pants hem (Table 3). The results of the size 16 live fit test showed that the least fit satisfaction was found in the underarm, neckline, abdomen, buttocks, hip girth, and thigh. More fit problems on the pants were found in size 16. Participant C, who spent more time and showed higher frequency in manipulation, provided more satisfactory fit except for the cross back, length of bodice, and pants (Table 3).

Table 3. Misses size 8 and Women's Plus size 16 fit model bodice and pant live fit evaluations from each participating student during live fitting session; comfort of clothing was evaluated on a scale of 1-5 with 1 being the least comfortable, 5 being most comfortable, and 3 being good fit; tightness of clothing was evaluated on levels of very tight, somewhat tight, good, somewhat loose, very loose.

\begin{tabular}{|c|c|c|c|c|c|c|}
\hline \multicolumn{7}{|c|}{ Size 8 Live Fit Evaluation } \\
\hline & \multicolumn{2}{|c|}{ Participant A } & \multicolumn{2}{|c|}{ Participant B } & \multicolumn{2}{|c|}{ Participant C } \\
\hline & Comfort & Tightness & Comfort & Tightness & Comfort & Tightness \\
\hline Bust girth & 4 & Good & 4 & Somewhat loose & 4 & Good \\
\hline Cross chest & 4 & Good & 5 & Good & 4 & Good \\
\hline Cross back & 4 & Good & 5 & Good & 2 & Very loose \\
\hline Underarm area & 3 & Somewhat tight & 3 & Somewhat tight & 4 & Good \\
\hline Neck front & 3 & Somewhat tight & 3 & Somewhat tight & 2 & Very tight \\
\hline Neck back & 3 & Very tight & 4 & Good & 2 & Very tight \\
\hline Neck side & 2 & Very tight & 3 & Somewhat tight & 2 & Very tight \\
\hline Waist girth & 4 & Good & 4 & Good & 1 & Very loose \\
\hline Length & 4 & Good & 4 & Good & 2 & Very short \\
\hline Abdomen & 4 & Good & 4 & Good & 5 & Good \\
\hline Buttocks & 5 & Good & 4 & Good & 5 & Good \\
\hline Hip girth & 4 & Good & 4 & Good & 2 & Somewhat loose \\
\hline Thigh & 3 & Somewhat loose & 4 & Good & 2 & Somewhat loose \\
\hline Crotch & 2 & Very loose & 2 & Very loose & 5 & Good \\
\hline Length & 2 & Somewhat short & 2 & Very short & 1 & Very short \\
\hline \multicolumn{7}{|c|}{ Size 16 Live Fit Evaluation } \\
\hline & \multicolumn{2}{|c|}{ PARTICIPANT A } & \multicolumn{2}{|c|}{ PARTICIPANT B } & \multicolumn{2}{|c|}{ PARTICIPANT C } \\
\hline & Comfort & Tightness & Comfort & Tightness & Comfort & Tightness \\
\hline Bust girth & 5 & Good & 5 & Good & 4 & Somewhat tight \\
\hline Cross chest & 5 & Good & 5 & Good & 3 & Somewhat tight \\
\hline Cross back & 5 & Good & 4 & Somewhat tight & 1 & Very tight \\
\hline Underarm area & 3 & Somewhat loose & 4 & Somewhat tight & 4 & Good \\
\hline Neck front & 3 & Somewhat loose & 4 & Somewhat tight & 5 & Good \\
\hline Neck back & 3 & Good & 4 & Somewhat tight & 5 & Good \\
\hline Neck side & 4 & Good & 2 & Very tight & 5 & Good \\
\hline Waist girth & 4 & Very loose & 5 & Good & 5 & Good \\
\hline Length & 5 & Good & 5 & Good & 3 & Very short \\
\hline Abdomen & 2 & Somewhat tight & 3 & Somewhat tight & 5 & Good \\
\hline Buttocks & 2 & Very tight & 3 & Very tight & 4 & Somewhat tight \\
\hline Hip girth & 2 & Very tight & 3 & Very tight & 5 & Good \\
\hline Thigh & 2 & Very tight & 3 & Very tight & 3 & Very tight \\
\hline Crotch & 5 & Good & 4 & Good & 5 & Good \\
\hline Length & 2 & Somewhat short & 5 & Good & 1 & Somewhat short \\
\hline
\end{tabular}


When comparing the virtual fitting result to the live fitting result using the same fit model, differences were apparent in the hem of the pants, wrinkle appearance, and bodice waist fit (Figure 4). The virtual fit model was shown with more wrinkles in the bodice and fewer wrinkles in the pants hip area. On the other hand, the live fit model appeared to have more wrinkles on pants and fewer wrinkles on bodice. This may be due to the difficulty in accurate depiction of tension or tightness of the garment to the avatar in the 3D CAD software. In examining the visual outcome of the two types of fitting, size 16 showed similar results as size 8 in that the wrinkle appearance and tension did not reflect each other in virtual and live fitting. Also, more fitting problems existed in the cross back area of the bodice, which may indicate the inappropriate pattern manipulation technique applied when adjusting armhole area.

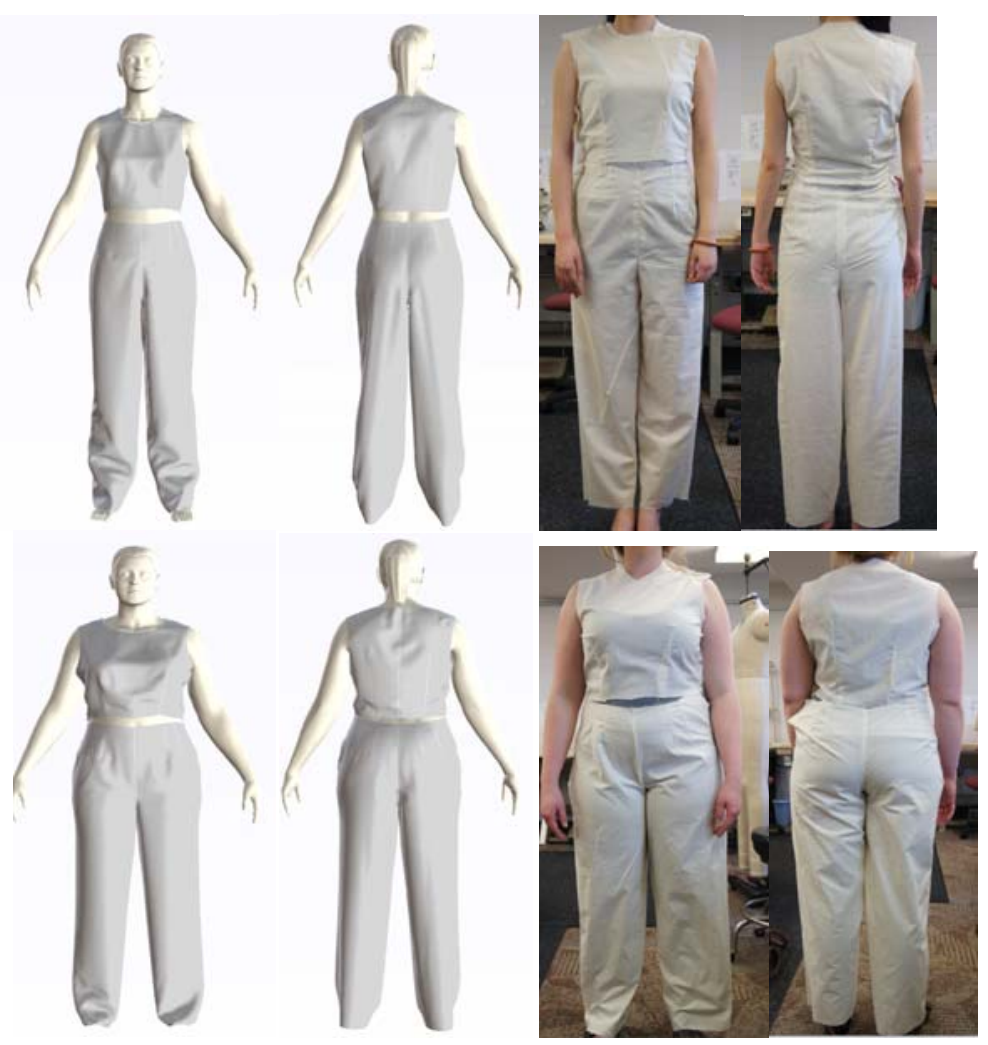

Figure 4. Fit testing on virtual model and live fit model for size 8 (up) size 16 (down).

\section{Conclusion}

The advantage of the virtual fit process is to increase efficiency in the product development process through providing an instant visual presentation of the garment on a virtual fit model.

The results indicate that participants spent more time on size 16 than size 8 , and twice as much time on the bodice than on the pants. Based on size 8 fit model evaluation, the model was least satisfied with the fit of the neckline, crotch, and pant hem. The results of the size 16 live fit test showed that poor fit was found in the underarm, neckline, abdomen, buttocks, hip girth, and thigh. It is possible that these problem areas were more challenging to the participants in understanding the 3D visual result of manipulation. The poor fit may have resulted from the visual barrier created by the 3D CAD software. In this study, the virtual fit model, created from the 3D body scanner, was not created with skin color, thus, convex and concave areas were less noticeable through the generic gray skin. Similarly, the garment was simulated in a gray color, which may reduce the visibility of clothing dimension and fitting ease. The visual presentation of the clothing on the virtual model may not be most ideal in presenting the fit of the garment. Participants had more difficulties in testing fit on the size 16 fit model than size 8 fit model. More fit problems on the pants were found in size 16 . This may be due to the lack of knowledge among participants concerning plus-size pants fit and pattern shape. It is possible that the order of operation in virtual fitting and pattern manipulation was appropriate for the better fit for plus-size fit model.

It is also important to note that although research has suggested that virtual fit process allows for accurate 3D depiction of the relationship between the garment and the body with minimal visual distraction [1], the 3D body scan may not be natural due to the standard scanning posture but is 
unique and historical in a sense [9]. Further, minor body movement and shadows may also create potential errors in the absolute accuracy of the scans [9].

However, there is a number of advantages in using the virtual fitting process. Participants were able to extract instant visual representation of the garment reflecting changes made in $2 \mathrm{D}$ pattern in the $3 \mathrm{D}$ CAD software. The 3D virtual fitting process can also offer much potential for one to explore in terms of tools. For instance, participant $\mathrm{C}$ spent more time during the simulation periods largely exploring available functions, such as changing the transparency of the fabric, to improve the visual accuracy of understanding the relationship between the garment and the virtual fit model. Although it is necessary to test an actual garment fit on certain body areas including neckline, underarm, and crotch, the 3D virtual fitting process would be useful and helpful in both teaching and industry application for obtaining instant visualization and gaining efficiency in the apparel product development process.

\section{References}

1. Ashdown, S., Loker, S., Schoefelder K., Lyman-Clarke L. (2004). "Using 3D scans for fit analysis", Journal of Textile and Apparel, Technology and Management, Vol. 4, No. 1, pp. 1-12.

2. Ashdown, S. and Dunne L. (2006). "A study of automated custom fit: Readiness of the technology for the apparel industry", Clothing \& Textiles Research Journal, Vol. 24, No. 2, pp. 121-136.

3. Bye, E., LaBat, K. L., Delong M. R. (2005). "An analysis of apparel industry fit sessions", Journal of Textile and Apparel, Technology and Management, Vol. 4, No. 3, pp. 1-5.

4. Song, H. K. \& Ashdown, S. (2010). "An exploratory study of the validity of visual fit assessment from three-dimensional scans", Clothing \& Textiles Research Journal, Vol. 28, No. 4, pp. 263278.

5. Cho, C., Park, J., Boeing, A., Hingston, P. (2010). "An implementation of a garment-fitting simulation system using laser scanned 3D body data", Computers in Industry. Vol. 61, pp. 550558.

6. Erwin, M., Kinchen, L., Peters, K. (1979). "Clothing for Moderns" (6th ed). Englewood Cliffs, NJ: Prentice Hall.

7. Bye, E., LaBat, K. L., Delong, M. R. (2006). "Analysis of body measurement systems for apparel", Clothing \& Textiles Research Journal, Vol. 24, No. 2, pp. 66-79.

8. Pandarum, R., Yu W., Hunter L. (2011). "3-D breast anthropometry of plus-sized women in South Africa", Ergonomics, Vol. 54, No. 9, pp. 866-875.

9. Apeagyei, P. (2010). "Application of 3D body scanning technology to human measurement for clothing fit", International Journal of Digital Content Technology and Its Applications, vol. 4, No. 7 , pp. $58-68$.

10. Stake, R. E. (1978). "Case study method in social inquiry", American Educational Research Association. Vol. 7, No. 2, pp. 5-8.

11. Stake, R. E. (1995). "The art of case study research", London, England: Sage Publication. 